Ophthalmologe 2014 · 111:817-818

DOI 10.1007/s00347-014-3081-5

Online publiziert: 11. September 2014

(c) Springer-Verlag Berlin Heidelberg 2014
P. Charbel Issa ${ }^{1}$ D. Pauleikhoff ${ }^{2}$ F.G. Holz ${ }^{1}$

${ }^{1}$ Universitäts-Augenklinik Bonn

2 St. Franziskus-Hospital, Münster

\title{
Teleangiektasien Typ 2
}

\section{Das internationale „MacTel Project"}

Im Jahr 2005 wurde das Macular Telangiectasia Project (MacTel Project; http:// www.mactelresearch.org) mit umfänglicher finanzieller Förderung durch die Lowy Foundation initiiert, um die klinische und grundlagenwissenschaftliche Forschung zu makulären Teleangiektasien Typ 2 zu befördern. Das MacTel Project machte sich zur Aufgabe, ein besseres Verständnis der klinischen Manifestationen und des natürlichen Verlaufs makulärer Teleangiektasien Typ 2 zu entwickeln, genetische Assoziationen aufzudecken und das Verständnis der pathogenetischen Mechanismen zu verbessern, um letztlich wirksame Therapien zu entwickeln.

Seit Beginn des Projektes hat sich die jährliche Publikationszahl zu makulären Teleangiektasien Typ 2 etwa verfünffacht, was die wissenschaftliche Aktivität und das rasch zunehmende Wissen um diese Erkrankung widerspiegelt. An dem internationalen Konsortium mit insgesamt 27 Zentren in 7 Ländern sind in Deutschland die Universitäts-Augenklinik Bonn und das St. Franziskus-Hospital Münster beteiligt.

Vor Beginn des MacTel Projects wurde die Erkrankung dieser auch als ,idiopathische juxtafoveoläre Teleangiektasien" und unter anderen Synonymen beschriebenen Veränderung als sehr selten angesehen. Mittlerweile wurde in einer populationsbasierten Studie hingegen eine relativ hohe Prävalenz von ca. 1:1000 in einer 43 bis 86 Jahre alten vorwiegend kaukasischen Population gefunden [3]. Es ist davon auszugehen, dass die Erkrankung tat- sächlich wesentlich häufiger ist, da diese Prävalenzberechnung lediglich auf der Auswertung von Fundusbildern basiert. Charakteristische morphologische Veränderungen können jedoch selbst bei asymptomatischen Patienten und/oder bei nahezu unauffälligem Fundusbefund mittels optischer Kohärenztomographie oder Fundusautofluoreszenz bereits früh detektiert werden [2]. Unter der Annahme einer ähnlichen Prävalenz in Deutschland wie in den USA wird die Zahl der Betroffenen bei ca. 43 Mio. Deutschen mit einem Alter $\geq 43$ Jahre (Quelle: Bevölkerungsstand 2010, Statistisches Bundesamt, www.destatis.de) auf mindestens 40.000 geschätzt.

》) Die Prävalenz ist wesentlich höher, als bisher angenommen

Aus klinischer Sicht ist eine gute phänotypische und funktionelle Charakterisierung der Erkrankung notwendig, um Patienten hinsichtlich ihrer Diagnose und Prognose zu informieren. Aufgrund des verstärkten wissenschaftlichen Interesses wurden in den letzten Jahren völlig neue morphologische und funktionelle Veränderungen beschrieben, die charakteristisch für makuläre Teleangiektasien Typ 2 sind. So zeigte sich beispielsweise mittels Fundusautofluoreszenz eine Reduktion des makulären Pigments im Bereich der betroffenen makulären Netzhaut, was neue Hinweise auf involvierte "pathways“ gab [4]. Gerade moderne bildgebende Verfahren erlauben nun eine klare klini- 
sche Abgrenzung zu anderen Erkrankungen, eine bessere Beurteilung des natürlichen Verlaufs und eine genauere Evaluation von aktuellen und zukünftigen Therapieansätzen. Aufgrund der neuen Erkenntnisse wird die Erkrankung mittlerweile als neurodegenerative Erkrankung der zentralen Netzhaut und damit als eine Makuladystrophie betrachtet, die erst sekundär mit den typischen vaskulären Veränderungen einhergeht. So können in frühen Stadien morphologisch fassbare Veränderungen noch ohne jede Gefäßbeteiligung detektiert werden.

In dieser Ausgabe des Ophthalmologen werden in einem ersten Beitrag die diagnostisch wegweisenden morphologischen Veränderungen makulärer Teleangiektasien Typ 2 dargestellt. In einem zweiten Artikel werden die funktionellen Implikationen beschrieben, deren Kenntnis die Sehbeschwerden bei den Betroffenen besser erklärt. Schließlich wird in einem dritten Beitrag eine Übersicht über therapeutische Ansätze gegeben. Auf andere, seltenere Formen idiopathischer makulärer Teleangiektasien (Typ 1 und 3 nach Gass und Blodi) wird außer in den differenzialdiagnostischen Erwägungen (s. Beitrag zu morphologischen Veränderungen) nicht näher eingegangen. Als weiterführende Literatur mit noch ausführlicherer Darstellung dürfen wir auf eine kürzlich publizierte umfängliche Übersichtsarbeit verweisen [1].
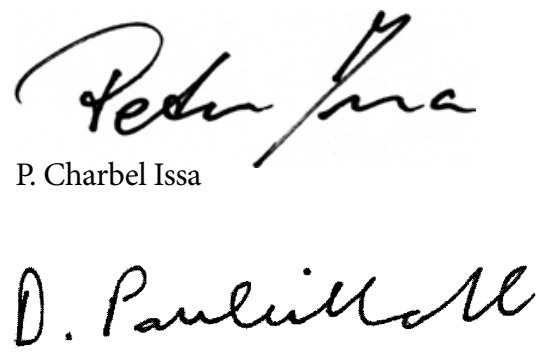

D. Pauleikhoff

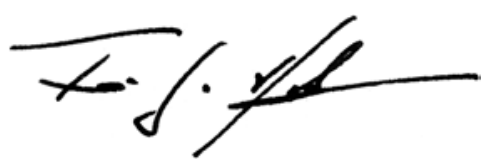

F.G. Holz

\section{Korrespondenzadresse}

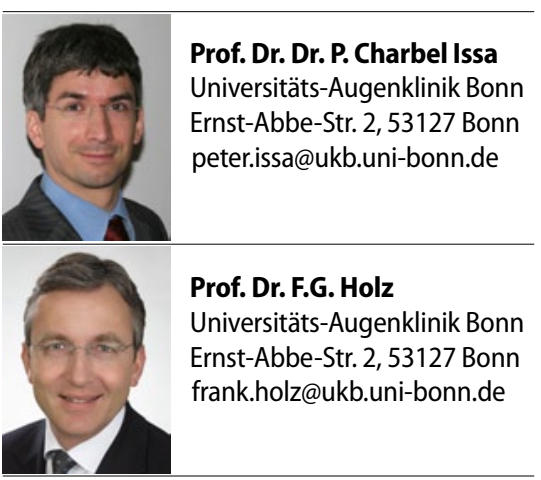

Interessenkonflikt. P. Charbel Issa, D. Pauleikhoff und F.G. Holz geben an, dass kein Interessenkonflikt besteht.

\section{Literatur}

1. Charbel Issa P, Gillies MC, Chew EY et al (2013) Macular telangiectasia type 2. Prog Retin Eye Res 34:49-77

2. Gillies MC, Zhu M, Chew EY et al (2009) Familial asymptomatic macular telangiectasia type 2 . Ophthalmology 116:2422-2429

3. Klein R, Blodi BA, Meuer SM et al (2010) The prevalence of macular telangiectasia type 2 in the Beaver Dam Eye Study. Am J Ophthalmol 150:55-62

4. Helb HM, Charbel Issa $P$, van der Veen RL et al (2008) Abnormal macular pigment distribution in type 2 idiopathic macular telangiectasia. Retina 28:808-816

\section{Kopfschmerzen durch 3-D}

Die Erzeugung von 3-D-Effekten über ein zweidimensionales Display erfolgt meist über das Prinzip der Stereoskopie. Dabei muss jeweils ein Seheindruck für das rechte und linke Auge separat dargestellt werden. Man spricht auch von Halbbildern. Die Separation der Halbbilder wird in Abhängigkeit von der Wiedergabemethode über verschiedene technologische Ansätze realisiert. Außerdem ist die Positionierung und perspektivische Variation der Halbbilder nötig. Dabei werden die Halbbilder horizontal versetzt zueinander dargeboten.

Sind diese Bedingungen erfüllt, setzen sich die Halbbilder über die kortikale Verarbeitung zu einem dreidimensionalen Gemeinschaftsbild zusammen. Ob der 3-DEffekt vor oder hinter der Displayebene wahrgenommen wird, hängt von der Zuordnung der Halbbilder zum jeweiligen Auge ab. Ist das rechte Halbbild dem rechten Auge und das linke dem linken Auge zuzuordnen, wird das Gemeinschaftsbild hinter der Displayfläche wahrgenommen, bei umgekehrter Zuordnung davor.

Durch die Art der Generierung des stereoskopischen 3-D-Effektes kann es beim Betrachter zu Problemen in der visuellen Verarbeitung kommen. Der sogenannte Vergenz-Akkommodation-Konflikt tritt in Erscheinung. Die Ebenen der Fokussierung und Fixation - gesteuert durch das visuelle System des Betrachters - fallen unnatürlicherweise nicht zusammen, sondern sind räumlich getrennt. Hierdurch wird das visuelle System übermäßig belastet. Dies kann zu Beschwerden wie ermüdeten Augen, Kopfschmerzen, Übelkeit, Schwindelgefühl, zur Wahrnehmung unscharfer Bilder und Doppelbilder führen.

Quelle: Ernst-Abbe-Fachhochschule Jena, www.fh-jena.de 